\title{
Current Levels of Drug Resistance Among Therapy-Naive HIV-infected Patients Have Significant Impact on Treatment Response
}

\author{
Inge Derdelinckx, MD*; Kristel Van Laethem, PhD*; Bart Maes, BSc*; Yoeri Schrooten, BSc*; Stéphane De \\ Wit, MD, PhD †; Eric Florence, MD, PhD ; Katrien Fransen, MScł; Sergio García Ribas, BSc $;$; Denise \\ Marissens, MSc† ; Michel Moutschen, MD, PhD§; Dolores Vaira, MSc§; Georges Zissis, MD, PhD†; Marc Van \\ Ranst, $\mathrm{MD}, \mathrm{PhD}^{*}$; Eric Van Wijngaerden, $\mathrm{MD}, \mathrm{PhD}^{*}$; Anne-Mieke Vandamme, $\mathrm{PhD}^{*}$
}

*REGA Institute for Medical Research and University Hospitals, Leuven;

$\dagger$ Universitaire Medisch Centrum, Sint-Pieter/Centre Hospitalier, Universitaire Saint-Pierre, Brussels;

Institute of Tropical Medicine, Antwerp;

$\S$ Centre Hospitalier Universitaire, Liège, Belgium

Resistant HIV can be transmitted from one patient to another. ${ }^{1}$ Recent large-scale European research found onetenth of viruses from untreated patients showing at least 1 resistance-related mutation. ${ }^{2}$ Such investigations are mainly driven by the concern that resistant virus could at some point hamper optimal treatment response. However, the impact of baseline drug resistance on treatment response is not well studied. Various genotypic interpretation methods are used to assess resistance in drug-naive patients. When transmitted resistance is the topic of interest, irrespective of treatment, primary mutations are considered most indicative. Secondary mutations could also be the result of natural variation. However, when interpreting resistance in view of the response to the installed treatment, all positions possibly contributing to the selective advantage of the virus in the presence of drug should be taken into account.

In this study protocol we wanted to document transmitted drug resistance among recently diagnosed therapynaive HIV-infected patients whose disease was diagnosed in 2000 in Belgium and to study therapy response considering transmitted drug resistance but also scoring the genotype according to interpretation algorithms for therapy response.

Samples from therapy-naive patients whose disease was diagnosed in 2000 were collected retrospectively through collaboration with 4 Belgian AIDS Reference Centers and Laboratories. All patients started antiviral therapy within 3 months of the baseline sample. The therapy remained unchanged for at least 2 months. Patients treated with non-approved antiretrovirals and patients judged to be noncompliant by the clinician were excluded.

Sequencing of the reverse transcriptase (RT) and protease (PRO) gene was performed. For the assessment of transmitted resistance, mutations were identified according to the list of the International AIDS Society (IAS)USA, including footnotes (March 2003). RT positions 44 and 118 and secondary PRO mutations were excluded since these were considered polymorphic sites. Further, mutations were interpreted with 2 genotypic resistance algorithms, the REGA algorithm version 5.5 (REGA, available through http://www.kuleuven.ac. $\mathrm{be} / \mathrm{rega/cev/links)}$ as well as the Stanford algorithm (НГV db, version 3.8.1, available through $\mathrm{http} / / /$ hivdb.stanford.edu). A value of $1,0.5$, or 0 was attributed to each drug in the prescribed regimen depending on a score of susceptible, reduced susceptible, or resistant, respectively. These values were summed in a susceptibility score (SS). The 5 categories of the Stanford algorithm were reduced to 3 by pooling the 2 highest and 2 lowest categories. CD4 count, log viral load (VL), and treatment changes were monitored at 3 (range 2-4) and 12 months after the start of treatment.

Therapy response was defined as a VL $<500$ copies/mL. Time to virologic success was defined as the time between the start of therapy and the first VL $<500$ copies/mL. Where appropriate, paired $t$-test, $\chi^{2}$ statistic, Fisher exact test, or odds ratio (OR) was used. Factors associated with therapy failure at 3 months were further analyzed in a multivariate logistic regression model. Proportions were reported with 95\% CI. $P$ values were 2-sided and considered significant at a level of $<0.05$. At 12 months, a complete case analysis (transmitted resistance), an intent-to-treat, and an on-treatment analysis were performed (SS). For the time-to-virologic-success analysis, group differences were assessed by the log-rank statistic. Data analysis was performed with SAS version 8.2 (SAS Institute, Cary, NC). 
Ninety-three patient samples were collected. Patients were predominantly male (58.1\%), black (51.6\%), and had mostly acquired HIV through heterosexual contact (58.1\%). Mean baseline CD4 count was 260.8 cells $/ \mathrm{mm}^{3}$ with a mean $\log$ VL of $5.1 \mathrm{log}$ copies/mL. Sequencing results were obtained for 80 samples for RT (86.0\%) and 83 samples for PRO (89.2\%). Genotypic evidence of transmitted resistance was detected in 6 patient samples $(6 / 83$, 7.2\%, 95\% CI: 3.4-14.9\%). In 4 samples this was nucleoside RT inhibitor (NRTI) related (4/80, 5\%, 95\% CI: 2.0-12.2\%); in 2 samples this was nonnucleoside RT inhibitor (NNRTI) related (2/80, 2.5\%, 95\% CI: 0.7-8.7\%); and in 1 sample this was PRO inhibitor (PI) related (1/83, 1.2\%, 95\% CI: 0.2-6.5\%). In 1 strain, NRTI and NNRTI resistance was combined (1/80, $1.3 \%, 95 \% \mathrm{CI}: 0.2-0.7 \%)$. The viruses carried the following mutations in RT: M41L + T215D/G + L210W; T69T/A/N/D; M184V; V108V/I; Y181C + 184V; and in PRO: L90M. Most viruses were carrier of at least 1 secondary PRO mutation(78/83 or $94.0 \%$ ), withamedian of 1 per sample (range $0-4)$.

Overall treatment response was good. At 2-4 months' follow-up, viral load was $<500$ copies $/ \mathrm{mL}$ in 71 patients $(71 / 80$ or $88.8 \%)$ with full resistance data available. At 12 months, 62 patients $(62 / 80$ or $77.5 \%)$ were considered responders, 9 patients $(9 / 80$ or $11.3 \%)$ had failed treatment. Nine $(9 / 80$ or $11.3 \%)$ had no data for this time point. In one-third of responding patients $(23 / 62$ or $37.1 \%)$, treatment had been changed due to reasons other than virologic failure. Patients with transmitted resistant virus were more likely to be nonresponders at 3 months. Of the 6 patients with transmitted resistance, only 2 were considered responders, compared with 69 of 74 without transmitted resistance (Fisher exact: $P=0.001)$. Other factors associated with treatment failure at this time point were $\log \mathrm{VL}(t$-test, $P=0.027)$ and CD4 count at treatment start $(t$-test, $P=0.008)$. In a multivariate logistic model, controlled for log VL and CD4 count at treatment start, the association of transmitted resistance with treatment failure remained significant (OR: 148, 95\% CI: 3.34->999.9, $P=0.027$ ). When considering the different drug classes separately, only transmitted NRTI resistance was significantly associated with therapy failure at 3 months (Fisher exact, $P=0.004$ ). After 12 months of treatment, 2 of 4 viruses with transmitted resistance and full data had failed treatment, and 2 responded ( 2 were lost to follow-up). At this time point, this association did not reach statistical significance.

Even though genotypic resistance profiles were not taken into account in the treatment choice, the majority of patients were treated only with drugs for which the virus was found fully susceptible, resulting in a maximal SS (REGA: 58/80 [72.5\%], HIVdb: 76/80 [95.0\%]). In the time-to-virologic-success analysis, we found patients with an SS of 2 as defined by HIVdb to have a longer time-to-virologic-success compared with all others (logrank, $P=0.032$ ). For REGA, both a cut-off of 2.0 and of 2.5 proved to be significant (log-rank, $P=0.032$ and $P$ $=0.029$, respectively). An SS of 2 as calculated with HIVdb and REGA was significantly associated with therapy failure at 3 months (Fisher exact, $P=0.032$ for both) and this remained significant in the multivariate logistic analysis, controlled for CD4 count and log VL at treatment start (OR: 125, 95\% CI 1.75->999.9, $P=$ 0.027). At 12 months of follow-up no significant association could be found between SS and treatment outcome.

In our dataset of 93 therapy-naive patients whose disease was diagnosed in Belgium in 2000, we evaluated transmitted resistance by counting only the major mutations reported in the IAS-USA list, thus excluding natural polymorphisms. This way, we found a prevalence of transmitted resistance of $7.2 \%$, which was not statistically different from the data reported in the combined analysis of resistance transmission over time of chronically and acute infected HIV-patients (CATCH) study, performed in 17 European countries including Belgium, where $9.6 \%$ of 1633 retrospectively collected samples were carriers of resistance. ${ }^{2}$

When evaluating the impact of resistance on first-line therapy response, we used 2 different types of analysis. First, we assessed whether transmitted resistance, scored as explained above, was associated with treatment response. Second, we did the same using an SS based on 2 different genotypic resistance algorithms for the interpretation of the detected mutations. We applied our in house-developed rule-based algorithm, REGA version 5.5, as well as HIVdb version 3.8.1, available through the Stanford website. The assessment of resistance in these different types of analysis overlapped partially. The number of viruses with transmitted resistance was higher compared with the number of viruses with a not-maximal SS as calculated with the HIVdb interpretation algorithm, since some patients carried viruses with resistance to drugs with which they had not been treated. Compared with HIVdb, the REGA algorithm v5.5 gave more weight to secondary mutations in PRO. As a result, 18 samples were additionally found to have a lower SS with the REGA algorithm, compared with the HIVdb algorithm.

We found resistance to have a significant effect on the time-to-virologic-success. This finding has been reported before for patients with primary or recent infection but not for chronically infected patients. ${ }^{3,4}$ Shorter time-tovirologic-success has been linked to more durable response to first treatment with highly active antiretroviral therapy $^{5}$ 
At 3 months, we found 2 predictors of virologic outcome: viruses with transmitted resistance, irrespective of the treatment, and viruses with an SS of 2, as calculated with REGA and HIVdb. These predictors remained significant in the multivariate analysis, controlled for CD4 and log VL at treatment start. None of these variables were predictive for therapy response at 12 months. Partly, this discordance can be explained by the fact that some nonresponding patients at 3 months later fully suppress the virus. Also, various other factors complicate the 12-month analysis. Treatment changes must be taken into account. Compliance and drug levels play a role, and these were not assessed here. To answer the long-term effects, both a larger sample and longer follow-up may be required.

When evaluating the drug classes separately, only transmitted NRTI resistance was significantly associated with treatment response. The absence of significance with the other classes could be due in part to lack of statistical power. NNRTI- and PI-related resistance was very low in this dataset, 2.5 and 1.2\%, respectively. Our findings stress the utmost importance of the knowledge of the resistance profile at baseline when choosing the NRTI backbone. Our data also argue against the use of a triple -NRTI combination as first regimen, but this regimen is no longer recommended in therapy-naive patients.

Duration of infection was not specified in this study. All patients had disease newly diagnosed in 2000 in Belgium and started immediate treatment. Considering the low mean CD4 count of 260 copies/mL, most patients were probably chronically infected. The data on the prevalence of resistance should therefore not be generalized for the entire drug-naive population but restricted to this subgroup. Also, it should be taken into account that inclusion was done retrospectively. Prospective and representative data should be collected, a process that is ongoing in Europe in the framework of the SPREAD (Strategy to Control Spread of HIV Drug Resistance) network (www.spread-europe.org).

\section{Acknowledgement:}

I. D. was supported by the Vlaams Instituut voor de bevordering van het wetenschappelijk-technologisch onderzoek in de industrie-IWT, Belgium; this work was in part supported by the AIDS Reference Laboratory of Leuven, which receives support from the Belgian Ministry of Social Affairs through a fund within the Health Insurance System, the Katholieke Universiteit Leuven (Grant nr. VIS/01/014), and the Fonds voor Wetenschappelijk Onderzoek Vlaanderen (G.0266.04); partial support was also received from Innogenetics NV, Ghent, Belgium.

\section{References}

1. Erice A, Mayers DL, Strike D, et al. Brief report: primary infection with zidovudine-resistant human immunodeficiency virus type 1 . $N$ Engl J Med. 1993;328:1163-1165.

2. Wensing AMJ, van de Vijver DAMC, Asjo B, et al. Analysis from more than 1600 newly diagnosed patients with HIV from 17 European countries shows that $10 \%$ of the patients carry primary drug-resistance: the CATCH study. In: Program and abstracts of the 2nd IAS Conference on HIV Pathogenesis and Treatment; July 13-16, 2003; Paris. Abstract LB1.

3. Little SJ, Holte S, Routy JP, et al. Antiretroviral-drug resistance among patients recently infected with HIV N Engl J Med. 2002;347: 385394.

4. Grant RM, Hecht FM, Warmerdam M, et al. Time trends in primary HIV-1 drug resistance among recently infected persons. JAMA. 2002; 288:181-188

5. Palella J, Chmiel JS, Moorman AC, et al. Durability and predictors of success of highly active antiretroviral therapy for ambulatory HIVinfected patients. AIDS. 2002;16:1617-1626. 